\title{
Diet and shopping habits of community-dwelling older adults during the COVID-19 pandemic: preliminary findings from the Hertfordshire cohort study
}

\author{
I. Bloom ${ }^{1,2}$, G. Bevilacqua ${ }^{1}$, J. Zhang ${ }^{1,2}$, W. Lawrence ${ }^{1,2}$, K.A. Ward ${ }^{1}$, C. Cooper ${ }^{1,3}$ and \\ E.M. Dennison ${ }^{1}$ \\ ${ }^{1}$ MRC Lifecourse Epidemiology Unit, University of Southampton, Southampton, UK, \\ ${ }^{2}$ NIHR Southampton Biomedical Research Centre, University of Southampton and University Hospital Southampton \\ NHS Foundation Trust, Southampton, UK and \\ ${ }^{3}$ NIHR Musculoskeletal Biomedical Research Unit, University of Oxford, Oxford, UK
}

The COVID-19 pandemic has led to dramatic changes in people's lives globally. Older adults have been especially vulnerable to adverse effects from the pandemic, and many have shielded. The pandemic and associated restrictions may serve to increase nutritional risk or exacerbate pre-existing malnutrition in older adults ${ }^{(1,2)}$. The aim of this study was to explore how community-living older people in the UK experienced the first wave of the COVID-19 pandemic and how it impacted their diets.

Participants drawn from the Hertfordshire Cohort Study, a pre-existing community dwelling cohort, were contacted by telephone and asked to complete a questionnaire administered by a trained researcher. Data collection took place from July to October 2020. Between March and October 2020, qualitative data were collected from a sub-set of these participants by conducting serial telephone interviews, with an interval of approximately three months. Discussions were audio-recorded, information related to the COVID-19 pandemic was transcribed verbatim and transcripts analysed thematically.

71 participants (39 men and 32 women), mean age 83.6 (SD 2.5) years, were surveyed. Over a third (37\%) of respondents lived alone. A large majority $(89 \%)$ of participants said their life was different compared to before the pandemic, with $80 \%$ reporting less social contact. However, 'healthiness' of diet and alcohol consumption were reported as generally unchanged over the timeframe surveyed. A sub-set of twelve participants were also included in the qualitative study ( 7 men and 5 women). Data from 35 qualitative interviews were used, comprising two or three timepoints per participant. Preliminary analysis identified three themes with relevance for participants' diets: shopping strategies and food accessibility, social and psychological repercussions, and coping strategies. Findings highlight some of the challenges associated with accessing shops and certain foods (e.g. fresh fruits and vegetables). Many participants received help with their shopping from family or neighbours; however, others continued to do their own shopping for a variety of reasons (e.g. not wanting to put others in danger, be a burden, or preferring to do their own shopping regardless). Social and psychological repercussions (e.g. loneliness and isolation, fear of the virus) appeared to link to a reprioritisation of behaviours (e.g. diet was deprioritised) for some participants. Some of the coping strategies used by participants included using meal delivery services and shopping at small convenience stores to avoid queueing at supermarkets.

Analysis is ongoing and will further aim to explore how older people's experiences and behaviours might have changed over the duration of the pandemic. The findings from this study could improve understanding of how community-living older adults could be supported to be more resilient in the face of a variety of changing circumstances that might impact their health and well-being.

\section{References}

1. Stratton R, Smith T \& Gabe S (2018) BAPEN.

2. Visser M, Volkert D, Corish C, et al. (2017) Nutr Bull 42, 178-186. 